\title{
Intelligence and Fuzzy Cognitive Maps: Scientific Issues, Challenges and Opportunities
}

\author{
Peter P. GROUMPOS \\ Department of Electrical and Computer Engineering Technology \\ University of Patras, Greece \\ groumpos@ece.upatras.gr
}

\begin{abstract}
Today humankind is facing an unprecedented set of many difficult problems and challenges that have never been encountered before. They urgently need realistic and long lasting solutions. Nowadays, practically all systems have become more and more complex and dynamic in nature. The modelling and controlling challenge of today's complex dynamic systems (CDS) is carefully reviewed. The fuzzy cognitive maps (FCM) approach is used to model CDS. Drawbacks and deficiencies of classical FCMs are identified and solutions to overcome them are mathematically developed. Two basic scientific fields are studied and analyzed on a joint synergistic way for the very first time. Intelligence and intelligent control (IC), cognition and cognitive control (CC) are two challenging scientific fields. A new modelling and control theory is proposed for addressing the complex dynamic systems (CDS).
\end{abstract}

Keywords: Modelling, Control, Complex dynamic systems (CDS), Intelligence, Cognition, Intelligent control, Cognitive control, Fuzzy logic, Fuzzy Cognitive Maps (FCMs).

\section{Introduction}

There is a saying which goes like this: "May you live in interesting times". It is widely reported as being an ancient Chinese curse but is neither Chinese nor ancient, it is recent and western. According to some historian this phrase was used in 1936 by Sir Austen Chamberlain, brother of the Prime Minister of that time. While purporting to be a blessing, this is in fact a curse. The expression is always used ironically, with the clear implication that 'uninteresting times', of peace and tranquility, are more life-enhancing than interesting ones. Like it or not we live in interesting times. They are times of danger and uncertainty; but they are also more open to the creative energy of men than any other time in history. We are certainly living through times of great anxiety not only on economic terms but also on many other aspects of our everyday life.

Nowadays, practical dynamic systems have become more and more complex $[16,31,34,40]$. The concept of complex dynamic systems (CDS) arises in many scientific fields, technological areas and everyday's problems [7,9,35]. Examples of these systems are: energy networks, storage and distribution, hybrid power systems with different renewable energy sources, robotics, health, artificial intelligence systems, safety and security systems, telecommunications, transportation networks, environmental systems, ecosystems, biological systems, social and economic systems, and many other scientific areas that can be considered to fall into the realm of complex dynamic systems (CDS). Such systems are often concurrent and hierarchically distributed, because they have to react to various kinds of events, signals, and conditions [31,34]. They are characterized by uncertainties, time delays, stochastic perturbations, fuzziness, complexity, hybrid dynamics, distributed dynamics and a large number of algebraic loops [30].The science of complex dynamical systems (CDS) is a multidisciplinary field aiming to undersatand the complex real world that surrounds us $[9,12$, $15,17,34,40]$.

Today the whole world is facing with an unprecedented set of problems that have never existed before [51]. Challenging and difficult problems such as Energy and Environment, Health and Ecology, Business and Economics, global poverty, food security, international stability, the future of the internet, and the ongoing process of social and cultural changes without knowing their long term effects to humankind. Some believe that we are steadily approaching the threshold of unimaginable chaos, calamity and destruction of our planet earth [7]. How are the international scientific communities approaching and study them wisely? There are various and lasting solutions to all these issues facing humankind. Most of them derive from the notion of the power of ideas so powerful that their effects upon the World will be most profound. Either we like or not the ideas and solutions come from the human brain [18]. The most powerful brain executives-attributes are Intelligence and Cognition $[5,10,14,26,52,53]$. We are eagerly 
searching for the relationship between Intelligence and Cognition by using the fundamental concept of "CONTROLS" [7,12]. The intelligent control (IC) and cognitive control (CC) are reviewed and a scientific approach is searched to see if and how they can be unified in a new control theory. The scope and impact of control systems could be substantially increased with the incorporation of properties we usually associate with intelligence, cognition, such as reasoning, planning, and learning [10,26,27,52-54].

In this paper the fundamental problem of modelling and controlling complex dynamic systems (CDS) is addressed for the first time using both basic scientific concepts: Intelligence and Cognition have been linked with the power of "CONTROLS" [7,12]. The fuzzy cognitive map concept, [30], was also used for the first time to link intelligence and cognition to solve problems of CDS. In section 2 the modelling problem of CDS is carefully reviewed and analyzed. The need to seek new advanced conceptual modelling methods is justified. In section 3 the interesting and challenging scientific topic of intelligence and intelligent control (IC) is briefly reviewed and it also presents the basics of IC. The only 30 years new approach of Fuzzy Cognitive Maps is provided in section 4 while section 5 gives illustrative examples. Section 6 identifies a number of drawbacks of FCM and provides some new and useful solutions to overcome them while section 7 provides for the first time a new definition of cognitive science using wisdom and fuzzy cognitive theories. A new unified control theory is provided and briefly analyzed for the first time using IC and $\mathrm{CC}$ in a synergistic way the: Intelligent Cognitive Unified Control (ICUC) theory. Section 8 gives some future research directions while section 9 provides some interesting and useful conclusions. An important remark: the topic of this paper is very broad, complex and difficult to be analyzed and presented in one single paper. Therefore in its current form, this study is not extensive and does not provide too many details. The interested reader can find many other sources.

\section{The Modelling Problem of Complex Dynamic Systems (CDS)}

Modeling is a fundamental and critical work which is always the starting point for the control, optimisation, and implementation of any physical and/or human made system. This is also the case for complex dynamic systems (CDS). However in the last 35-40 years CDS presented many problems both in mathematical modelling, control implementation and philosophical foundations. Complex dynamic systems (CDS) comprise collections of many heterogeneous entities which interact with other entities and their environment which usually has a lot of uncertainties, fuzziness, ambiguities and structural complexities. Interactions among subsystems are localized, often seeking autonomy and self-organizing, while most of the times are nonlinear, dynamic, fuzzy and possibly chaotic. Thus the study of CDS presents a new challenge for the academic and scientific communities that investigate how relationships between parts of CDS give rise to the collective behaviors of a "system" and how it interacts and forms relationships with its environment. Today's most CDS have some specific characteristics, among which: uniqueness, weak structuredness of knowledge about the system, incompleteness of its dynamic behavior, antagonism among different agents, the composite nature of system, heterogeneity of elements composing the system. Furthermore decisions must be made ensuring the smooth, reliable, stable and cost effective operation of each of the subsystem as well as of the whole CDS.

Another important feature of CDS is that a network structure, including hierarchical one, self-organization can amount to: (1) disconnecting certain constituent nodes from the system, (2) connecting previously disconnected nodes to the same or to other nodes, (3) acquiring new nodes, (4) discarding existing nodes, (5) acquiring new links, (6) discarding existing links, (7) removing or modifying existing links. In addition today's society's challenging problems demand CDS to have a number of PropertiesAbilities (P-A) such as; co-evolution, anticipation, adaptation, cooperation such as swarming, intelligence, consciousness, cascading failures in electrical grid, hurricanes and self-repairing materials, cognition and wisdom.

All these collective dynamics of a CDS give rise to the so called: Emergent Evolution PropertiesAbilities (E.E.P-A) at higher scales in space and/ 
or time. Under such conditions, the key problem of complex dynamic systems and control theory consists in the development of methods of qualitative analysis of the dynamics and behavior of such systems and in the construction of efficient control algorithms for their efficient operation. In a general case, the purpose of control is to bring the system to a point of its phase space which corresponds to maximal or minimal value of the chosen efficiency criterion [15-18]. One more critical aspect that must be seriously taken into consideration is that the human presence in all CDS is inevitable. This problem is very critical in studying CDS because we are actually dealing with Dynamic Systems and want to understand their long-term qualitative behavior. However the focus is not on finding precise solutions to the equations, which most of the times are not well mathematically defining the complex dynamic system. Such a search is often hopeless. The solutions been sought would rather answer some questions like "Will the CDS settle down to a steady state in the long term, and if so, what are the possible steady states?" The hope should be that the steady state will be well defined. Does the long-term behavior of the system depend on its initial condition? or "does the past history of the behavior of the CDS influences its long-term behavior" or "what is more important: correlation or causality between the states"?

Therefore the modeling and analysis of CDS in the presence of uncertainty, fuzziness, ambiguity and of principally non-formalizable problems and not probable of having strict and precise mathematical formulation of the system. On environments where decisions are semi- structured or unstructured, complex dynamic systems (CDS) need to be readdressed. All above characteristics must be taken into consideration. Construction of models of CDS must be based on the use of experts and their extensive knowledge about the CDS. This knowledge should be wisely used. Thus modeling CDSs is indeed a real chalenge. It is not a straightforward and an easy task. It is a difficult and challenging exercise which cannot be studied sufficiently using today's models and methods. Therefore we need to seek new advanced conceptual modelling methods. The human factor cannot be ignored and thus intelligence and cognition do play a critical and fundamental role in modelling and controlling today's CDS.

\section{Intelligence and Intelligent Control (IC)}

What is intelligence? There are a few ways to think about "intelligence". Each one approaches this fundamental question depending on his/her personality as well as the particular situation. One definition by the Merriam Webster dictionary: "the ability to learn or understand things or to deal with new or difficult situations". For centuries, philosophers have tried to pinpoint the true measure of intelligence. Socrates said, "I know that I am intelligent, because I know that I know nothing" while Einstein said, "The true sign of intelligence is not knowledge but imagination" and another time "Logic will get you from A to Z . Imagination will take you everywhere".

Everybody agrees that there isn't even a consensus as to what constitutes intelligence in the first place. It is widely accepted that there are different types of intelligence - analytic, linguistic and emotional, to name a few-but psychologists and neuroscientists disagree over whether these intelligences are linked or whether they exist independently from one another.

Although the issue of what constitutes intelligence is not clear yet, scientists have moved to define mathematically the concept of Intelligent control (IC). And this, because as the need to model and control complex dynamic systems (CDS) increases, it is important to look beyond engineering and computer science areas for control strategies to see if we can learn from them. For example, humans have the capacity to receive and process enormous amount of sensory information from the environment, exhibiting integrated sensorimotor intelligence as early as two years old [19]. The fact is that there are problems of control which cannot be formulated and studied in the conventional differential/difference equation mathematical framework. To address these problems in a systematic way, a number of methods have been developed that are collectively known as intelligent control (IC) methodologies. Consequently, the field of Intelligent Control (IC) has been emerged. Intelligent control (IC) is a class of control techniques that use various computing approaches like neural networks, fuzzy logic, machine learning, evolutionary computation and 


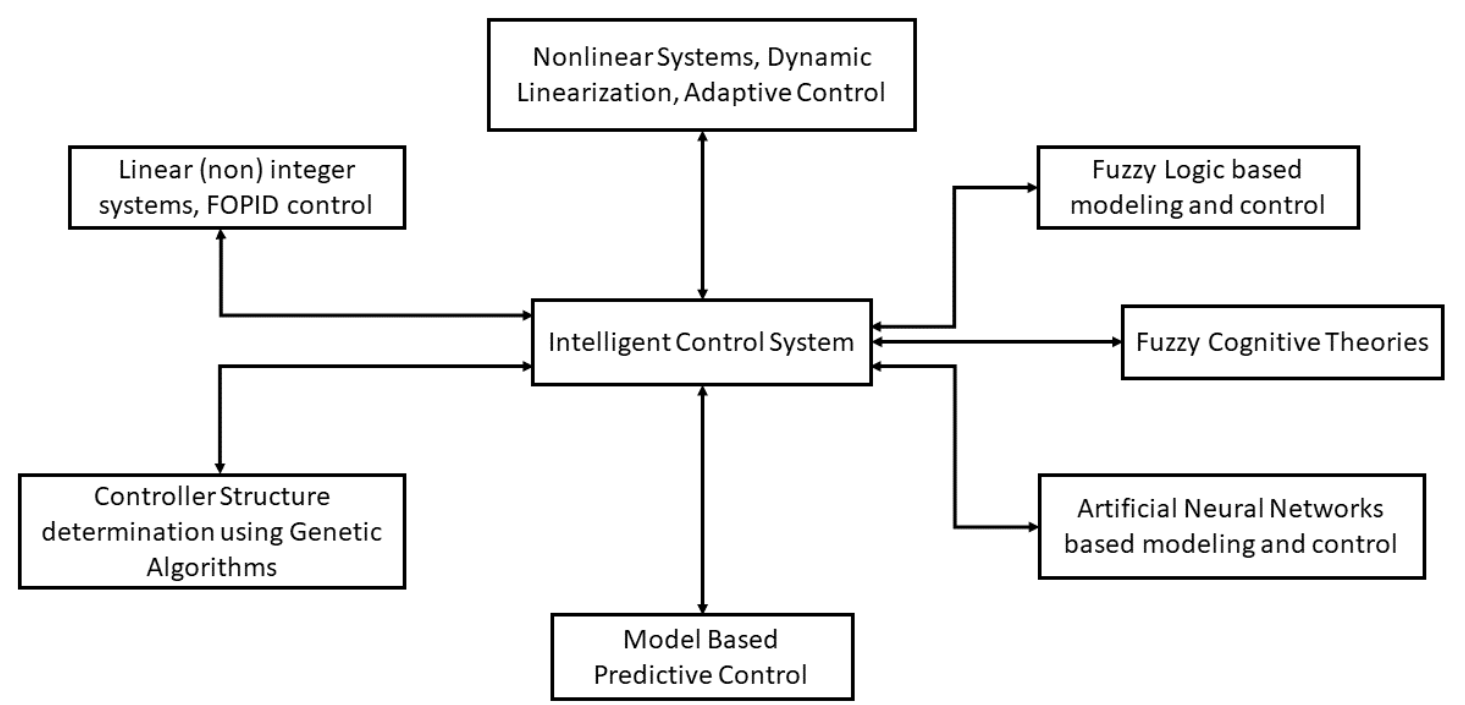

Figure 1. A representative diagram of Intelligent Control (IC)

genetic algorithms [5]. A simple but not complete "form" of IC is shown in Figure 1. In the minds of many people, particularly outside the control area, the term" intelligent control" has come to mean some form of control using fuzzy and/or neural network methodologies. However, IC does not restrict itself only to those methodologies.

There are significant differences between conventional and intelligent control (IC). It is worth remembering at this point that intelligent control (IC) uses conventional control methods to solve "lower level" control problems and that conventional control is included in the area of intelligent control. Intelligent control attempts to build upon and enhance the conventional control methodologies to solve new challenging control problems. The word control in "intelligent control" has different and more general meaning than the word control in "conventional control". First, the processes of interest are more general and may be described, for example by either discrete event system models or differential/difference equation models or both. This has led to the development of theories for hybrid control systems that study the control of continuous- state dynamic processes by discrete-state sequential machines. In addition to the more general processes considered in intelligent control (IC), the control objectives can also be more general. Intelligent control can be divided into the following major sub-domains: (partial list) 1) Neural network control, 2) Machine learning control 3) Fuzzy Control, 4) Bayesian control, 5) Intelligent agents, 6) Genetic Control, 7) Neuro- fuzzy control, 8) Soft computing control, 9) Conscious control 10) Fuzzy Cognitive control, 11) Expert Systems.

For all these different Intelligent Control (IC) subfields there have been many books and research articles covering extensively most of them. $[5,7,10,52,53]$. Intelligent control - control based on fuzzy logic and neural networks - has changed the face of industrial control engineering whether in terms of autonomous spacecraft operation, exploratory robots or increasing the profitability of mineral- processing or steelrolling plants. In addition, new intelligent control methods and techniques are created continuously as new models of intelligent behavior are created and computational methods are developed to support them. In addition, the field of cognitive science and cognitive control (CC) has emerged and challenges in a synergistic way the problem of modelling and controlling CDS only from the IC point of view and especially from the scientific area of Artificial Intelligence (AI). It is important to note here that the extremely interest scientific topic of AI cannot be covered within the scope of this paper. However it should be stressed that tech giants are investing billions in transformative technologies in order to get a lead over each other 
in AI. The West's largest tech firms, including Alphabet (Google's parent), Amazon, IBM, Apple, Facebook, and Microsoft are investing huge sums to develop their AI capabilities, as their counterparts in China are doing. Although it is difficult to separate tech firms' investments in AI from other kinds, so far in 2017 companies globally have completed around $\$ 21.3 \mathrm{bn}$ in mergers and acquisitions related to AI, according to PitchBook, a data provider, or around 26 times more than in 2015. Projects like the Deepmind of Google, the Watson of IBM, the Siri of Apple, BAIDU commonly known as the "Google of China", the Google Blue Brian, are among the many projects which have been undertaken. This paper concentrates more on the problems that AI, IC and CC can jointly investigate. There is a need to study and investigate the solutions to this problem while all three different generic scientific fields, IC, AI and CC are considered in a synergetic approach. One methodology that can meet this objective is represented by the Fuzzy Cognitive Maps (FCM) theories and methods.

\section{Fuzzy Cognitive Maps Intelligent Control}

\subsection{Introductory Remarks}

Fuzzy Cognitive Maps (FCMs)[6,30] is a new methodology for modeling complex dynamic systems (CDS) and has been around only for the last 25-30 years $[8,20,21,43,46]$. FCMs basically exploit the knowledge and experience of "people". Fuzzy Cognitive Maps came as a combination of the methods of fuzzy logic and neural networks. They constitute a computational method that is able to examine situations during which the human thinking process involves fuzzy and/or uncertain descriptions. They are the evolution of the Cognitive Maps which were introduced by Axelrod [6]. Fuzzy Cognitive Maps (FCMs) were first introduced by Kosko in 1986 [30]. They are a soft computing methodology which gives users the ability to encounter problems in the same way the human mind does; using a conceptual procedure which can include ambiguous or fuzzy descriptions [21,30,47,54]. Therefore, FCMs offer a simple, fast, flexible, economical, and versatile approach to a variety of problems (engineering, health, environmental, economic, mechanical, business, tourism, political, social) which are extremely complex and a purely mathematical approach would be time consuming, laborious and require wasting many resources without finding meaningful and realistic solutions at the end. Kosko [30] introduced FCMs 30 years ago as a method to represent the causal relationship between concepts, variables, states, nodes and constraints. Their goal is to represent knowledge in a symbolic way and model the behavior of systems containing elements with complex relationships, which sometimes can be hidden or illegible [54].

\subsection{Why Use Fuzzy Cognitive Maps?}

Studying very carefully and at the same time wisely the above two sections the need to search for new innovative and advance mathematical models was obvious. There are six main reasons that suggest and require the utilization of Fuzzy Cognitive Maps (FCMs) in modelling and controlling complex dynamic systems (CDS): 1) Complexity 2) Nonlinearities 3) Uncertainty 4) chaos 5) Ambiguity and 6) Fuzziness.

The majority of the real world systems include these six properties-parameters. The conventional control methods for such systems cannot confront these properties-parameters as the FCMs can do. Furthermore the two basic controls: Intelligent and Cognitive have not been used in a joint effort but rather separately [27]. Thus, FCMs are about to play a major role in the future regarding the modeling, analysis, design and control of complex dynamic systems (CDS) by using the IC and $\mathrm{CC}$ in a cooperative and synergistic approach. However this must be accomplished in a careful and systematic way.

\subsection{Brief Historical Remarks}

A historical link of FCM theories is connected to graph theory and goes back to the 18th century. As said FCMs are directed graphs, or digraphs, and thus they have their historical origins in graph theory. Informally a graph is a set of nodes joined by a set of lines or arrows. Graph theory is the study of graphs, mathematical structures used to model pair wise relations objects from a certain collection [11]. A graph is thus a context which refers to a collection of vertices or nodes and a collection of edges that connect pairs of vertices. The paper written by Leonard Euler on 


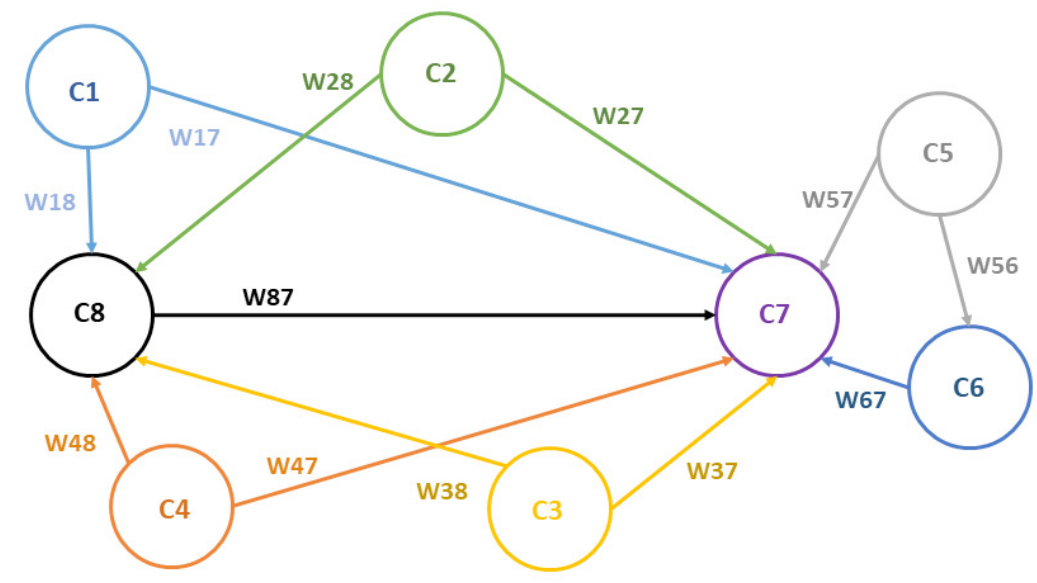

Figure 2. A Representative Fuzzy Cognitive Map Diagram

the Seven Bridges of Konigsberg and published in 1736 [11] is regarded as the first paper in the graph theory. Graphs are among the most ubiquitous models of both natural and humanmade structures. For many centuries ideas now embodied in graph theory have been implicit in lay discussions of networks $[11,18,32]$. The explicit linking of graph theory and network analysis began only in 1953 and has been rediscovered many times since. Analysts have taken from graph theory mainly concepts and terminology; its theorems, though potentially valuable for the analysis of real data, are generally neglected. Today Network analysts thus make too little use of the graph theory.

However, till today, they have been used to model many types of relations and process dynamics in physical, networks, engineering, biological, health, energy and social systems. Surprisingly, graphs have not been used, almost at all, on economic and business systems. Political scientist Robert Axelrod [7] was the first to use digraphs to show causal relationship among variables as defined and described by people, rather than by the researcher. Axelrod called these diagraphs Cognitive Maps (CM). Many studies have used CM to look at decision-making as well as to examine people's perceptions of complex social systems. Kosko, modified Axelrods CMs, which were binary, by applying fuzzy causal functions with real numbers in $[-1,1]$ to the connections, thus the term Fuzzy Cognitive Maps (FCM) [30]. Kosko was also the first to model FCMs and to compute the outcome of a FCM, or the FCM inference, as well as to model the effect of different policy options using a neural network computational method [30].

\subsection{Mathematical Foundations}

A FCM presents a graphical representation used to describe the cause and effect relations between nodes, thus giving us the opportunity to describe the behavior of a complex dynamic system exhibiting on or more than one of the above mentioned properties-parameters a)-e) of CDSs, in a simple and symbolic way. In order to ensure the operation of the system, FCMs embody the accumulated knowledge and experience from experts who know how the system behaves in different circumstances over a long time span. In other words they recommend a modeling process consisting of an array of interconnected and interdependent nodes $\mathrm{Ci}$ (variables), as well as the relationships between them $\mathrm{W}$ (weights). Concepts take values in the interval $[0,1]$ and weights belong in the interval $[-1,1]$. Figure 2 shows a representative diagram of a Fuzzy Cognitive Map (FCM).

The sign of each weight represents the type of influence between concepts. There are three types of interconnections between two concepts $\mathrm{Ci}$ and $\mathrm{Cj}$ :

- wij $>0$, an increase or decrease in $\mathrm{Ci}$ causes the same result in concept $\mathrm{Cj}$.

- $\quad w i j<0$, an increase or decrease in $\mathrm{Ci}$ causes the opposite result in $\mathrm{Cj}$.

- $\quad w i j=0$, there is no interaction between concepts $\mathrm{Ci}$ and $\mathrm{Cj}$.

The degree of influence between the two concepts is indicated by the absolute value of wij. The value of each concept at every simulation step is calculated, computing the influence of the 
interconnected concepts to the specific concept, by applying the following calculation rule:

$$
A_{i}[k+1]=f\left(k_{2} A_{i}[k]+k_{1} \sum_{j=1, j \neq i}^{N}\left(A_{j}[k] w_{j i}\right)\right)
$$

where $\mathrm{k}$ represents time, $\mathbf{N}$ is the total number of concepts and

- $\mathrm{Ai}^{(\mathrm{k}+1)}$ : the value of the concept $\mathrm{C}_{\mathrm{i}}$ at the iteration step $\mathrm{k}+1$

- $\mathrm{Ai}^{(\mathrm{k})}$ : the value of the concept $\mathrm{C}_{\mathrm{j}}$ at the iteration step $\mathrm{k}$

- Wij: the weight of interconnection from concept $\mathrm{Ci}$ to concept $\mathrm{Cj}$

- $\mathrm{k} 1$ : the influence of the interconnected concepts in the configuration of the new value of the concept $A_{i}$

- $\mathrm{k} 2$ : the proportion of the contribution of the previous value of the concept in the computation of the new value

- $\quad$ f: the sigmoid function

$f=\frac{1}{1+e^{-\lambda x}}$

where $\lambda>0$ determines the steepness of function f. The FCM's concepts are given some initial values which are then changed depending on the weights; the way the concepts affect each other. The calculations stop when a steady state is achieved, the concepts' values become stable. In most applications $\mathrm{k} 1$ and $\mathrm{k} 2$ are set equal to one (1).

One major drawback of the early FCM approach has been the convergence problem of the algorithms. Given the values of the initial values of the weights at least two problems have been observed: 1) always the final values of the weights converge to the same value regardless the original conditions of the system and 2) in some cases the algorithms do not converge at a final steady state value. In order to overcome these two convergence problems learning algorithms are used. The main ideas stem from neural networks. Unsupervised methods such as Hebban techniques are the most common used. More specifically Nonlinear Hebbian Learning (NHL) has been used to overcome partially this drawback $[4,10,41]$.
In this learning algorithm the nodes are triggered simultaneously and interact in the same iteration step with their values to be updated through this process of interaction. The algorithm which modifies the initial weights defined by experts is described by the following relationship:

$w_{i j}[k+1]=g \cdot w_{i j}[k]+h \cdot A_{j}[k]\left(A_{i}[k]-\operatorname{sgn}\left[w_{i j}\right] \cdot w_{i j}[k] A_{j}[k]\right)$

where, the coefficient $g$ is called weight reduction learning parameter and the coefficient $h$ is a very small positive scalar factor also called learning parameter. The "learning parameters" $g$ and $h$ of the above equation are very important and they usually take values between $g \ni[0.9,1]$ $g \ni[0.9,1]$ and $\mathrm{h} \ni[0,0.1] \ni[0,0.1]$. The weights $\mathrm{w}_{\mathrm{ij}}$ are updated for each iteration step for all $\mathrm{N}$ concepts and they are used in equation (1) in order to compute the new values of concepts. Two stopping criteria terminate the procedure. The first one concerns the minimization of function $\mathrm{F}_{1}$ which is the sum of the square differences between each Desired Output Concept i (DOC $)$ and a target value $T_{i} T_{i}$ is defined as the mean value of the range of $\mathrm{DOC}_{\mathrm{i}}=\left[\mathrm{T}_{\mathrm{i}}^{\mathrm{min}}, \mathrm{T}_{\mathrm{i}}^{\mathrm{max}}\right]$.

$F_{1}=\sqrt{\sum_{i=1}^{m}\left(D O C_{i}-T_{i}\right)^{2}}$

$T_{i}=\frac{T_{i}^{\min }+T_{i}^{\max }}{2}$

The second criterion is the minimization of the variation of two subsequent values of Desired Output Concepts:

$F_{2}=\left|D O C_{i}^{(k+1)}-D O C_{i}^{(k)}\right|$

When the termination conditions are met the new final weight matrix $\mathrm{w}_{\mathrm{ij}}$ with the DOCs are returned. More on other drawbacks and proposed solutions of the up today theories of FCMs are given in the next section. A more comprehensive mathematical presentation of FCMs theories, methods and algorithms is provided in $[1,4,20,22,28,41,43,44,45]$.

\section{Illustrative Examples}

FCM theories have been around for only 30 years. However they have been used extensively sometimes obtaining excellent results and some other times not as satisfactory been expected. Due to space limitations only one illustrative example is given. However the reader interested 


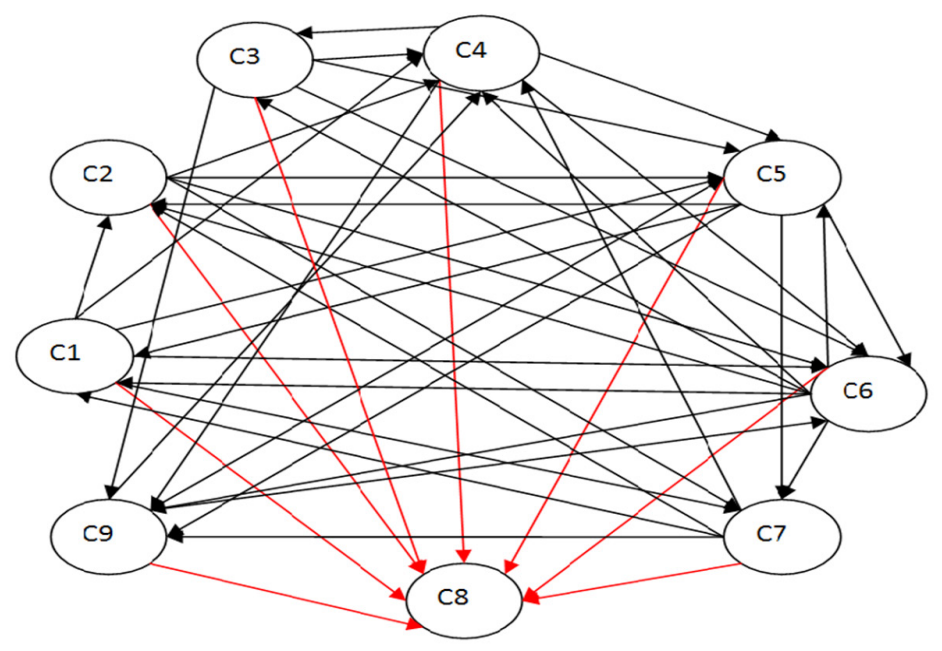

Figure 3. A conceptual FCM model for Stability of the Enterprise

in this subject is encouraged to carefully study $[2,4,8,20,21,23,33,36,39,42-46,49,50]$ and many more from the open source literature.

\subsection{The Illustrative Example: Decision Making in Stability of an Enterprise in a Crisis Period Using FCMs}

A simple example of Decision Making for the Stability of an Enterprise in a Crisis Period using FCMs can show that the new approach of FCMs in modelling CDS is very promising. In the current FCM model there is only one decision concept (output), i.e. the stability of an enterprise in a crisis period is studied. The factor concepts are considered as measurements (via special statistic research) that determine how each measurement-concept will function in this model. They are the following: $\mathrm{C} 1$ : sales, $\mathrm{C} 2$ : turnover, C3: expenditures, C4: debts \& loans, C5: research \& innovation, C6: investments, C7: market share, C8: stability of enterprise and C9: present capital; is the output of the system.

Figure 3 shows a simple FCM model for the enterprise system. At this point it should be noted that in economic systems we can't talk about causality but only for correlation between the defined factor-concepts of this problem. Experts noted that the acceptable-desired region for the final value of concept $\mathrm{C} 8$ is:

$0.70 \leq C_{\mathbf{8}}^{(\text {final })} \leq 0.95$

If $C_{\mathbf{8}}^{(\text {final) }}$ is inside this region then we can say with great certainty that the enterprise is out of danger and the economic crisis period does not put at risk the stability and the smooth function of the enterprise. Weights in Table 1 are determined after defuzzifying (with COA method) the fuzzy values that were given from the experts (mostly economists).

In addition, the degree of occurrence of each input-concept factor is denoted with qualitative degrees of high, medium, and low. Respectively for the output concept $\mathrm{C} 8$ the qualitative degrees are very low, low, medium, high and very high.

Table 2. Initial factor-concepts fuzzy value

\begin{tabular}{|c|c|}
\hline Factor-concepts & Case 1 \\
\hline C1 & H \\
\hline C2 & M \\
\hline C3 & L \\
\hline C4 & L \\
\hline C5 6 & M \\
\hline C6 7 & L \\
\hline C9 & L \\
\hline
\end{tabular}

The initial values of the outputs were set equal to zero.

Table 3. Final decision-concepts

\begin{tabular}{|c|c|}
\hline Decision-concepts & Case 1 \\
\hline $\begin{array}{c}\text { C8 (Stability of the } \\
\text { Enterprise) }\end{array}$ & $\mathbf{0 . 8 3 9 1}$ \\
\hline
\end{tabular}

The iterative procedure ends when the values of $\mathrm{Ci}$ concepts make no difference between the latest three iterations. Considering $\lambda=1$ for the unipolar sigmoid function and after 11 iteration steps the FCM reaches an equilibrium point.

We considered initial values for the concepts:

$\mathrm{A}^{(0)}=\left[\begin{array}{lll}0.8867 & 0.46670 .09670 .46670 .09670 .09670 .650 .4667\end{array}\right]$

$\mathrm{A}^{(11)}=\left[\begin{array}{l}0.81400 .87080 .71450 .61210 .47430 .74620 .85810 .83910 .4779\end{array}\right]$ 
Table 1. Weights between concepts for Enterprise System

\begin{tabular}{|c|c|c|c|c|c|c|c|c|c|}
\hline & C1 & C2 & C3 & C4 & C5 & C6 & C7 & C8 & C9 \\
\hline C1 & 0 & 0.6 & 0 & -0.4 & 0.2 & 0.3 & 0.6 & 0.8 & 0 \\
\hline C2 & 0 & 0 & 0 & -0.2 & 0.2 & 0.5 & 0.1 & 0.3 & 0 \\
\hline C3 & 0 & 0 & 0 & 0.4 & -0.5 & -0.4 & 0 & -0.6 & -0.5 \\
\hline C4 & 0 & 0 & -0.4 & 0 & -0.7 & -0.8 & 0 & -0.7 & -0.4 \\
\hline C5 & 0.2 & 0.3 & 0 & 0 & 0 & 0.5 & 0.3 & 0.2 & -0.2 \\
\hline C6 & 0.3 & 0.2 & 0.6 & 0.5 & -0.3 & 0 & 0.3 & 0.3 & -0.4 \\
\hline C7 & 0.4 & 0.3 & 0 & -0.2 & 0 & 0 & 0 & 0.4 & 0.5 \\
\hline C8 & 0 & 0 & 0 & 0 & 0 & 0 & 0 & 0 & 0 \\
\hline C9 & 0 & 0 & 0 & -0.3 & 0.2 & 0.4 & 0 & 0.2 & 0 \\
\hline
\end{tabular}

It is observed that in the latest three iterations there is no difference between the values of concepts $\mathrm{Ci}$. So after 11 iteration steps, the FCM reaches an equilibrium point where the values do not change any more from their previous ones, that is:

Since the final value of $\mathrm{C}_{8}^{(\text {final })}$ is inside the acceptable region, defined by the experts, then it could be assumed with great certainty that the enterprise can survive the crisis period. This example is very timely given the world economic uncertainties.

\section{Advanced Fuzzy Cognitive Maps Models}

Despite the many theoretical developments of FCMs based on the material presented on the previous section 4 and their application successes (references given in section 5) FCMs still have a number of drawbacks and deficiencies. All theories are essentially based on the material of section 4 will be referred as the "classical FCM theories". They do not go into the depth of the dynamic behaviour of complex systems. In addition the initial system structure described by experts and the learning principles-algorithms cannot follow the evolution of the complex dynamic system (CDS). The natural-human world evolves and the human-made systems progress by applying knowledge derived from observations of and familiarity with repeatable events and phenomena of nature. Our perceptions, understanding and ability to model the real world enable us to develop policies, processes and products that are invented and required by applying various technologies. Generally speaking, our goal is either to control if possible

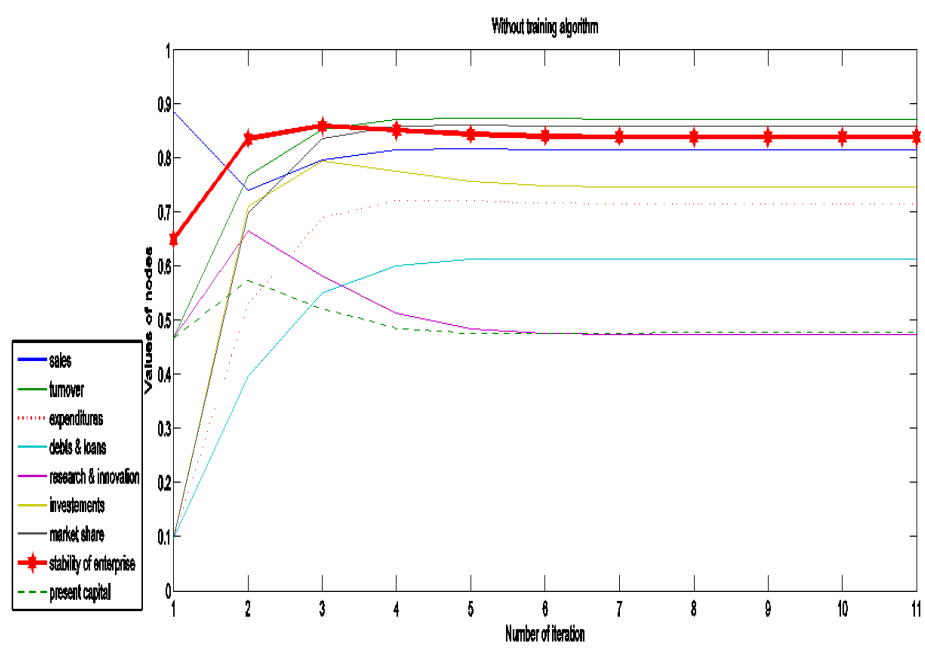

Figure 4. Subsequent values of concepts till convergence 
optimally (and exploit) the natural phenomena, or to create human-made systems and processes with the desired properties. However this is not possible using the "classical FCM theories". The main problem stems from the existing approach having all $\mathrm{N}$ concepts in one vector variable. The values $A_{i}$ of all $N$ concepts at all instant of times, $k$, are calculating eq.1. Similarly the learning -training algorithms for upgrading the causality coefficients Wij, is done for all $\mathrm{N}$ concepts and for all instant of times, $\mathrm{k}$, using equation 3. However this is not mathematically correct as it is shown below.

One major drawback is that all concepts, $\mathrm{C}$, (say $\mathrm{N}$ ) are grouped as one vector and every calculation of their values A, (iteration time k, eq.1) regarding the behavior of the complex dynamic system, here, equations 1 and 3, is performed for every concept. This is not mathematically correct since from the total number $\mathrm{N}$ of concepts, there is a number of concepts whose values remain constant for long period of time when other concepts are changing slowly or fast. In this classical FCM formulation another problem has been that no matter what initial conditions were used, the algorithm was always converted to the same final values $[20,24,38,42,48]$.

Another drawback has to do with the NHL learning method (Eq.3). While running several simulations we have observed that due to the way weights are being calculated if the number of iterations of the algorithm is increased, in order to reach a steady state, the causality sometimes reverses all or some of the Wij values. This is a very serious drawback as it changes the causality between concepts and in several occasions instead of having a lower we are going to have a larger result which can cause serious problems not only in the interpretation of the obtained results but also on stability issues to a number of real life systems. This is also not mathematically correct and needs further investigation after the separation of the total $\mathrm{N}$ concepts into states, inputs and outputs as it is shown below.

For the above raised drawbacks some solutions and explanations must be given. The research team of the Laboratory for Automation and Robotics under the supervision of the author of this paper has provided some interesting and valuable solutions. As it was mentioned, above in this section, in the classic FCM representation ALL the concepts
$(\mathrm{N})$ are ALL the parameters which are going to be examined regardless their nature. However, in a CDS, even when it is described in a fuzzy way through an FCM the main concept is the same. Each system has its states, inputs, outputs and other parameters and constraints. However, since an FCM is a representation of such a system, this fundamental characteristic should be taken into consideration. For this reason, as in the classic control theory methods $[9,12,17,35]$ the concepts of a Fuzzy Cognitive Map (FCM) are separated into the following three categories:

a) Fuzzy State Concepts: The concepts describing the dynamic behavior of the system, $x$

b) Fuzzy Input Concepts: The inputs of the system, $u$

c) Fuzzy Output Concepts: The concepts describing the outputs of the system, $y$

This separation of the N FCM concepts into the above a)-c) categories, was introduced for the first time by the author [24,31].

This separation also provides a very clear and useful presentation through the state space approach [12]. This can also be seen clearly in Figure 5.

In this way a better knowledge of the dynamic behavior of the CDS can be gained. The proposed separation facilitates not only the understanding of the system's operation but also the calculation of the concepts' values in their physical nature as the states, inputs and outputs of the real system. Then in a second effort [37-39], a revised approach in modelling Fuzzy Cognitive Maps was presented in detail. The new model was composed by the following equations:

$$
\begin{aligned}
& x[k+1]=x[k]+\frac{\Delta x[k+1]}{\sum_{j=1, j \neq i}^{n}\left|w_{j i}\right|} \\
& y[k+1]=y[k]+\frac{\Delta y[k+1]}{\sum_{j=1, j \neq i}^{n}\left|w_{j i}\right|} \\
& \Delta x_{k+1}=A \Delta x_{k}+B \Delta u_{k} \\
& \Delta y_{k}=C \Delta x_{k}+D \Delta u_{k}
\end{aligned}
$$




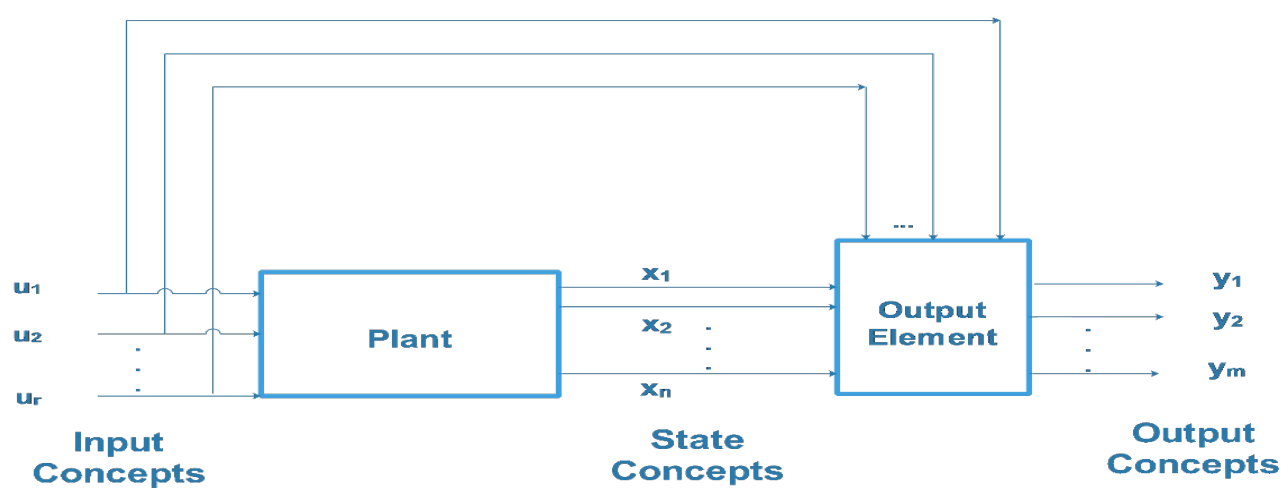

Figure 5. The proposed State space FCM model

where $\mathrm{x}_{\mathrm{k}}$ are the "concept states" and, $\mathrm{u}_{\mathrm{k}}$ the "concept inputs" and $\mathrm{y}_{\mathrm{k}}$ the "concept outputs". They were used to calculate the variation caused by the change in the input and state concepts to the state and output concepts at each time step $(\mathrm{k})$.

In this representation $\mathrm{A}, \mathrm{B}, \mathrm{C}$ and $\mathrm{D}$ are individual weight matrices derived from the initial weights defined by the experts [4]. Each weight matrix has the appropriate dimensions depending on the a)-c) categories of the total number $\mathrm{N}$ of concepts. The elements of A depend on the states weights and the elements of B show how each input concept affects the state concepts of the system. Matrix $\mathrm{C}$ shows how the output concepts are related to the state concepts, $x(k)$ and $D$ shows how the input concepts could directly affect the output $\mathrm{y}(\mathrm{k})$ concepts. In the same paper a new sigmoid function $f$, instead of eq. 2, is proposed [37] which is:

$f(x)=m+\frac{M-m}{1+e^{\left(-r\left(x-t_{o}\right)\right.}}$

Another attempt to address some drawbacks of today 's FCMs theories and methods has been made in, (Vergini and Groumpos 2017) [48]. After the implementation of the classical FCM method on various applications and without using any learning algorithm, it has been observed that for a FCM with determined and constant weight matrix the use of Eq.1, Eq.4 and Eq.5 leads to the same output value no matter what the initial concept values are. Starting from this observation, and looking for a solution to this problem, it was determined that, apart from the initial given concepts' values, the initial disturbances are necessary in order to calculate more accurate the system output. A different disturbance of the given initial conditions would force the system to reach a different equilibrium point because it has a different impact on the system response. This gives a partial but very useful solution to the more than 30 years of the classical FCMs methods in which no matter what initial conditions were given the solution was converging to the same final condition. This is not acceptable on modern systems approach.

Taking the above reasoning as a basic idea, the classical FCM equation, Eq.1, and the weight values' explanation a new method was proposed in the paper, [48]. It was proposed that "If $\mathrm{w}_{\mathrm{ji}}>$ 0 this means that an increase of the $\mathrm{Cj}$ value will cause an increase of $\mathrm{Ci}$ value." This means that in order to have a change in the $\mathrm{Ci}$ concept value, there should be a disturbance on the value of $\mathrm{Cj}$, in other words there should be a change, $\mathrm{DCj}$. The new model suggested to be used in order to calculate the new concept values, $\mathbf{A}$ is given in details in [48]:

$A_{i}(k+1)=A_{i}(k)+\Sigma[W j i D A j(k)]$

where

$D A j(k)=A j(k)-A j(k-1)$

In the same paper [48], the sigmoid problem was also studied and a new sigmoid function was proposed which is the same of the study [37] and its equation (11), given above was first proposed by Mpelogianni and Groumpos [3739]. Please note that in classical FCM studies all $\mathrm{N}$ concepts remain in one and the same vector. However, in the studies [4], [24-25], [39] and [48] the concepts of a FCM are separated for the first time to state concepts $\mathrm{x}$, input concepts 
$\mathrm{u}$ and output concepts $\mathrm{y}$. This becomes clearer in the study of (Anninou et. al.2017) [4]. In [4] the problem of separating the concepts into states, inputs and outputs is addressed in more details. This was actually dictated by the medical expertise since eq. 1 could not address the complex knee injured problem in which all variables were put into one vector with all concepts been treated exactly the same. This could not be accepted by the physicians. As a result of this, research investigation created a new approach of Dynamic Fuzzy Cognitive Knowledge Networks (DFCKN) and is presented here again.

The two equations extracted from the classic FCM are the following:

$$
\begin{aligned}
& x(k+1)=f[A x(k)+B u(k)] \\
& y(k)=f[C x(k)+D u(k)]
\end{aligned}
$$

where $x(k) \in R^{n}$ is the concept state vector, $u(k)$ $\in \mathrm{R}^{\mathrm{r}}$ is an exogenous known concept input vector, $\mathrm{y}(\mathrm{k}) \in \mathrm{R}^{\mathrm{m}}$ is the concept output vector and $\mathrm{f}$, as before, is an activation function. For $\mathrm{f}$, eq. 2 can be used. The new model (eqs. 14 and 15) was implemented for first time in diagnosing meniscus injury in IFAC World Congress 2017 with very encouraging results [4]. This is an evolutionary type of Fuzzy Cognitive Maps (FCM) that arose from the need for updating classic methodology in order to overcome its drawbacks, concerning the single calculation rule equation (1), updating the weights, equation (3), stability and other real-time problems. The new proposed DFCKN model is able to diagnose knee injuries and specifically meniscus injuries in a very simple way and to distinguish between acute and degenerative injury. This new approach was tested for its usefulness in Decision Support Systems (DSS) in medicine by considering 17 real cases of patients. Subsequently we observe the evolution of the injury by administering a proposed treatment by the physician. Results of this new method, which are presented in detail, in [4], are very satisfactory for both two levels and treatment stage, and in total agreement with Magnetic Resonance Imaging (MRI) outcomes. The results were considered very good by the physicians and thus we claim that our results are very good. The whole methodology is the outcome of a close collaboration between engineers and medical doctors and is significant because it is a promising tool which sets aside the main disadvantages of Fuzzy Cognitive Maps and allows us a wide-range use in many real-time problems. These results and the previous ones reported earlier in a number of papers have given us the right to refer the new proposed theories as Advanced FCMs theories in contrast to the classical FCMs theories which basically use the early methodologies $[1,6,20,30,41-43,46]$.

\section{Cognitive Science and Cognitive Control}

Cognitive science is the interdisciplinary, scientific study of the mind and its processes. It examines the nature, the tasks, and the functions of cognition (in a broad sense). Cognitive scientists study intelligence and behavior, with a focus on how nervous systems represent, process, and transform information $[14,19,29]$. Mental faculties of concern to cognitive scientists include: language, memory, attention, perception, emotion and reasoning [50]. To understand these faculties, cognitive scientists borrow from fields such as: artificial intelligence, fuzzy theory, linguistics, psychology, philosophy, deep learning, wise learning, neuroscience, and anthropology $[35,54]$. The typical analysis of cognitive science spans many levels of organization, from learning and decision to logic and planning; from neural circuitry to modular brain organization. All these can be achieved thanks to digital computers. However the question still remains what digital computer can and cannot do [13]. The fundamental concept of cognitive science is that "thinking can best be understood in terms of representational structures in the mind and computational procedures that operate on those structures" $[19,35]$. The cognitive sciences began as an intellectual movement in the 1950s often referred to as the "cognitive revolution" [35]. Since then theorists have proposed many accounts of the conceptual system. The dominant theory, however, has been the semantic memory view, which arises from a proposed distinction between semantic and episodic memory. However, all related studies have as base the physiological aspects especially, the psychological process of language comprehension has received considerable attention. A number of widereaching theories of text comprehension have been developed. However cognitive therapies were forced based on their clinical practices and/ or their personal lives because formal cognitive 
theory grounded solely in psychological science was, and remains, sufficiently disorganized not to be of much assistance [35]. This approach of thinking about the conceptual system remains dominant even today. Researchers throughout the cognitive sciences continue to adopt various forms of semantic memory models in their working accounts of the cognitive system. A problem seems here that the current practice is not using the most basic function of the human brain that of learning. Learning is perhaps the most generic psychological principle and has received an enormous amount of empirical support from highly controlled laboratory conditions in a wide variety of species regarding a broad range of behaviors. Our educational system is based upon the principle that people learn. Therapists of cognitive problems have observed that all psychological theories of psychopathology maintain that learning plays an etiological role in psychological and behavioral disorders and that all persons who benefit from psychological interventions learn something [29]. It now seems somewhat ironic that Kazdin's [29] criticizes that behavior therapy was not based on modern learning theory. Indeed, there are many new developments on other related scientific fields that must be taken into consideration when studying cognitive science. A new scientific "cognitive science" has been developed for the first time and is shown here in Figure 6. In this new "cognitive concept" additional concepts of wisdom, cognition and fuzzy cognitive theories (FCT) are considered for the first time. This has c direct impact in developing the Cognitive Contro] (CC) theory.

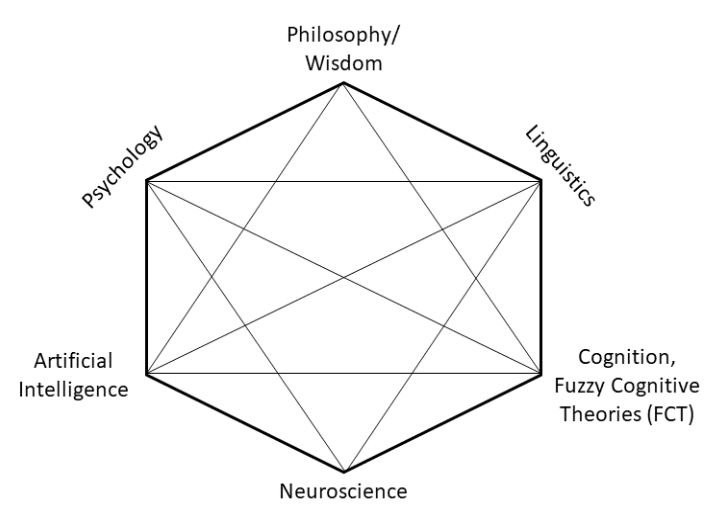

Figure 6. Fields that contribute to the new "cognitive science".

The broad goal of cognitive science is to characterize the nature of human knowledge - its forms and content - and how that knowledge is used, processed, and acquired. In other words, scientifically Cognitive Control (CC) must be clearly defined and mathematically well formulated taking into consideration theories and issues that are outside the physiological and psychological sciences. Cognitive Control (CC) allows one's mind to override his/her impulses and helps to make decisions based on his/her goals, rather than his/her habits or reactions. Cognitive Control (CC) is one's mind's ability to actively create an information picture that will guide his/her behavior. This is what allows him/her to select a certain behavior that was accepted as appropriate or reject a behavior that he/she decided to be inappropriate. Cognitive Control (CC) is at the center of one's mind self-awareness, his/her highest level of consciousness, and one's mind willpower. A simple schematic diagram of Cognitive control is given in Figure 7. Everybody believes that the incorporation of properties has been usually associated with cognition - including reasoning, planning, and learning - within control systems holds the promise of greatly expanding the scope and impact of the field. These arguments suggest that the controls community should takealeadership roleinshaping the future cognitive control research agenda. Again due to space limitation this topic of Cognitive Control (CC) is not covered scientifically as properly as possible.

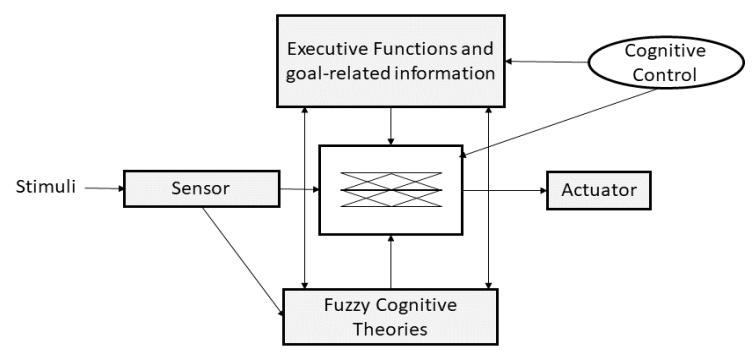

Figure 7. A simple diagram of Cognitive Control (CC).

The most powerful brain executives- attributes are Intelligence and Cognition as they were analyzed above. As a result of this there are two powerful methods: the IC and the CC. Now having reviewed these two fundamental control concepts a basic question arises: how Intelligent Control (IC) and Cognitive Control (CC) can be related to each other? The humankind problems are given and well known. Both approaches must and are addressing these same problems! What is 
their role in solving them? Can they complement each other and together provide valuable, viable and lasting solutions to the so many problems been faced by humankind? The answer can only be positive.

However, many engineers and scientists from both scientific fields disagree that the natural and mathematical derived methods and tools of Intelligent Control (IC) and Cognitive Control (CC) can work together in a harmonious and synergistic way to address today's global problems. They are almost isolated on their own field or cave and they hardly talk to each other. The only wise solution to this problem is to propose to both scientific communities to work together and jointly to formulate a new scientific field of Intelligent Cognitive Unified Control (ICUC). This new scientific theory is proposed here as one generic and realistic solution although is still a theoretical approach. It is shown in the self explained diagram of Figure 8. Everything starts with the physical and/or human made world. All problems and challenges from this physical nature as well as the human made world must be analyzed, studied and comprehend by both fields of intelligent control (IC) as well as by the cognitive control (CC). Each scientific field would use the scientific and mathematical theories, methods, techniques and corresponding tools depending to the particular case to generate the associated "true knowledge". Knowing the "true knowledge" for the specific problem using the intelligence outcome and the cognitive process a unified action would be decided to be the intelligent cognitive unified control (ICUC) theory. Now based on this a wise and useful lasting solution can be developed for the specific problem. A strong mathematical foundation is needed that will bring together existing methods and techniques from both fields of IC and CC as well as create new innovative concepts and methods.

Finally what would be the realistic and useful solutions to the global and regional problems? It is proposed to modify the classical theme to: think globally, act locally and learn wisely fast (TG. $\boldsymbol{A} \boldsymbol{L} . \boldsymbol{L} \boldsymbol{W}$.). . This approach could give humankind what could be called "realistic best practices". A realistic best practice can be defined as a project, product, service or organization that presents an innovative approach that has proven effectiveness, with visible, measurable results, is accessible to the public, and can be replicated by others.

\section{Future Research}

After reading carefully this paper, the reader may find that the list of future research directions is numerous, quite open and very challenging. There is no doubt that future scientific generations will confront and take up this challenge. They will use Artificial intelligence (AI), Intelligent Control (IC) and Cognitive Control (CC) in a harmonious and synergistic approach to develop a global coherence and sustainable growth for the humankind.

Each scientific topic will have its own list.

A. For AI include:

- Machine learning (ML). ML is so popular that it has become synonymous with AI. The researchers are now focusing on scaling the state-of-the-art ML algorithms to large datasets. New Data Mining (DM) algorithms are needed for analyzing and processing Big Data structures.

- Deep Learning (DL) is re-branding of neural networks- a class of models inspired by biological neurons in our brain. DL has been driving force for lots of applications in AI like object recognition, speech, language translation, playing computer games and controlling self driving cars. New methods and algorithms independent of $\mathrm{AI}$ and ML are needed.

- Internet of Things (IoT) is a concept that daily use physical devices are connected to the internet and can communicate with each other via exchange of data. The data collected could be processed intelligently to make the devices smarter.

- Neuromorphic computing relies on neurons based models. Research is needed to develop hardware chips that can directly implement neural network architecture. Neuromorphic chips are needed so that data is both processed and stored in the chip in an analog manner and can generate synapses when required, saving time and energy.

- Other topics without giving more details are: mechatronics and/or robotics, Algorithmic game theory, Computational mechanism designs, computer vision, Reinforcement Learning (RL), Natural Language Processing (NLP), algorithms for the Big Data Driven World (BDDW) and theories of Cyber physical space (CPS) systems. 


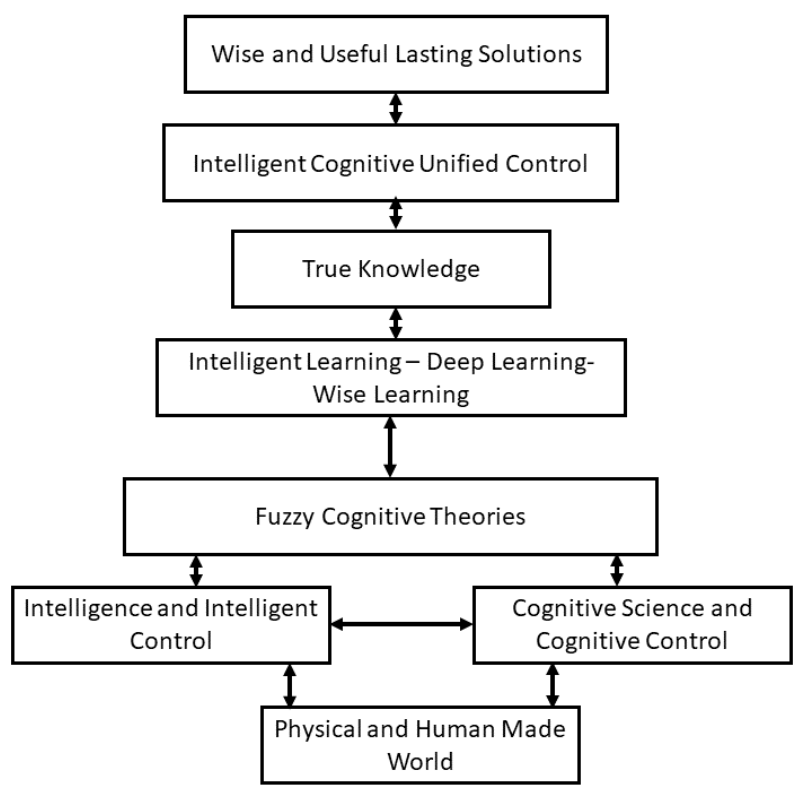

Figure 8. The proposed Intelligent Cognitive Unified Control (ICUC) structure

B. For the advanced FCM and Intelligent Control (IC) include:

- Develop further the new advanced models for FCM

- Study the development of the causality weights $\mathrm{W}_{\mathrm{ij}}$ using learning method and investigate their relationship to probability correlation coefficients.

- Define a new approach in developing the IC using the new advanced FCM theories

- Develop new software tools for various CDS and perform extensive simulations for real life problems.

- Perform simulation studies for real life problems using the classical FCM methods and the advanced FCM theories. Compare the results and identify which of the two methods overcome the drawbacks and deficiencies when modelling and controlling CDS.

C. For CC include:

- Control strategies for the adaptive management of cognitive resources in real-time systems need to be developed. Cognitive control systems will need to aggregate and consolidate information, balance long-term and immediate priorities, and shift attention dynamically as circumstances dictate.
- Human operators are still the preferred recourse for responding to rare and sudden adverse events. Research is needed to develop automationsystemsthatcan exhibit humanlike capabilities in such situations.

- Modeling and estimation take on added dimensions in cognitive control, with representations of self, the environment, objectives, and other elements required. Such representations must often be developed from partial and uncertain information.

Finally the most challenging future research topics are related to the proposed Intelligent Cognitive Unified Control (ICUC) of the Figure 8.

\section{Conclusions}

In this paper the fundamental and difficult problem of modelling and controlling complex dynamic systems (CDS) is addressed for the first time using two basic scientific concepts: Intelligence and Cognition have been linked with the power of "CONTROLS". The analysis and efficient control of CDS are impossible without a formal model of the system. However today's' technologies for building such models for CDS are not sufficient. Qualitative description of most of the parameters of complex dynamic systems (CDS) results inevitably in fuzziness, complexity, ambiguity and uncertainty. One of the challenges of accepting the "operation" of any complex dynamic system is 
the ability to make Decisions so the system runs efficiently and cost effectively. It was shown that new conceptual and innovative approaches are needed. One new approach has been introduced only 30 years ago. This is the fuzzy cognitive map (FCM) approach. However it is shown that FCM as have been formulated in all these years have many drawbacks and deficiencies. In this paper certain specific answers have been given to overcome these drawbacks. New advanced FCM methods are given which have been developed by the research team leaded by the author.

Intelligence and Intelligent Control (IC) along with the powerful Cognitive Science and Cognitive Control (CC) can indeed provide useful, realistic and lasting solutions to the problems of the present society. Till today both scientific fields have developed excellent theories, techniques and tool each one on its own field. Both are addressing most of the same global and regional problems. Both are developing solutions for the same problems. However they do not cooperate and complement each other. They must lead to many and endless discussions and provide some realistic and solid scientific solutions to problems that humankind is facing. Solutions

\section{REFERENCES}

1. Aguilar J. (2005). A survey about fuzzy cognitive maps papers (Invited paper), Int. J. Comput. Cog, 3(27).

2. Anninou, A. P., Groumpos P. P. \& Polychronopoulos, P. (2013). Modeling health diseases using competitive fuzzy cognitive maps, Artificial Intelligence Applications and Innovations, 88-95. Springer, Berlin Heidelberg.

3. Anninou, A. P. \& Groumpos, P. P (2013). Non Linear Hebbian Learning Techniques and Fuzzy Cognitive Maps in Modeling the Parkinson's Disease. In $21^{\text {st }}$ Mediterranean Conference on Control and Automation MED'13, Chania (pp.709-715). IEEE.

4. Anninou, A. P, Groumpos, P. P., Poulios, P. \& Gkliatis, I. (2017). A New Approach of Dynamic Fuzzy Cognitive Knowledge Networks in Modelling and Diagnosing Process of Meniscus. In The 20th World Congress of the IFAC, Toulouse, France, July 2017 (pp. 6035-6040). from IC and CC are waiting to help us. Creative and gentle hearts along with wise minds should recognize that there are solutions from combing IC and CC. A new unifying theory is needed. This is proposed as the Intelligent Cognitive Unified Control (ICUC) theory. It will systematically and scientifically search mathematical foundations in order to investigate, analyze and provide solutions to the problems that the world is facing. This new proposed theory is absolutely necessary to accept that "True Knowledge" is the one and only one that can lead humankind to developing models and creating the ICUC theory. This "True Knowledge" must come from more than one expert who has extensive experience in observing and working on today's CDS. There is no doubt that Figure 8 and the new proposed ICUC theory will be developed someday for the good benefit of the society and the humankind. For the future generations. A Greek poet has said: "our today's actions will be judged by the future unborn generations". In closing, the proposed new and innovative structure for the ICUC can be used by governments, leading global organizations, academic and research communities to carry out extensive studies for most of the challenges and problems that humankind is facing.

5. Antsaklis, P. J. \& Passino, K. M. (ed.) (1993). An Introduction to Intelligent and Autonomous Control. Kluwer Academic Publishers.

6. Axelrod, R. (1976). Structure of Decision: The Cognitive Maps of Political Elites. Princeton University Press, Princeton, NJ.

7. Azar, T. A. \& Vaidyanathan, S. (2016). Advances in chaos theory and intelligent control. Springer.

8. Bardossy, A. \& Duckstein, L. (1995). Fuzzy rule-based modeling with applications to geophysical, biological, and engineering systems. CRC.

9. Bar-Yam, Y. (1997). Dynamics of Complex Systems. Addison-Wesley, Reading, Massachusetts.

10. Behera, L. \& Kar, I. (2010). Intelligent Systems and Control: principles and applications. ACM Digital Library. 
11. Biggs, N., Lloyd, K. E. \& Wilson, J. R. (1976). Graph Theory, 1736-1936. Oxford University Press.

12. Dorf, R. C. \& Bishop, R. H. (2017). Modern control systems, $13^{\text {th }}$ Edition. Pearson.

13. Dreyfus, H. (1979). What Computers Can't Do. New York: MIT Press.

14. Dreyfus, H. \& Dreyfus, S. Mind over Machine: The Power of Human Intuition and Expertise in the Era of the Computer. Oxford, U.K., Blackwell.

15. Filip, F. G., Zamfirescu, C. B. \& Ciurea, C. (2017). Computer-Supported Collaborative Decision-Making, Part of the Automation, Collaboration, \& E-Services book series (ACES, volume 4). Springer.

16. Filip, F. G. \& Leiviskä, K. (2009). LargeScale Complex Systems. In: Nof, S. (eds), Springer Handbook of Automation. Springer, Berlin, Heidelberg.

17. Filip, F. G. (2012). A Decision-Making Perspective for Designing and Building Information Systems, Int. J. of Comp. Comm. \& Control, 7(2), 264-272.

18. Gauchy, L. A. (1813). Recherche sur les polydres - premier mmoire, Journal de l'Ecole Polytechnique, 9(16), 66-86.

19. Gazzaniga, S. M. (2002). Cognitive Neuroscience. The Biology of the Mind, $2^{\text {nd }}$ ed., 615. Norton \& Co., New York.

20. Glykas, M. (2010). Fuzzy Cognitive Maps: Advances in Theory, Methodologies, Tools and Applications. Berlin, Heidelberg: Springer-Verlag Berlin Heidelberg.

21. Groumpos, P. P. \& Stylios, C. D. (2000). Modeling supervisory control systems using fuzzy cognitive maps, Chaos Solitons \& Fractals, 113, 329-336.

22. Groumpos, P. P. (2010). Fuzzy Cognitive Maps: Basic Theories and Their application to Complex Systems. In book: Glykas, M. (Ed) and preface by Kosko, B., Fuzzy Cognitive Maps: Advances in Theory, Methodologies, Tools and Applications, 1-22. Springer.

23. Groumpos, P. P. \& Anninou, A. P. (2012). A theoretical mathematical modeling of Parkinson's disease using Fuzzy
Cognitive Maps. In $12^{\text {th }}$ Intern. Confer. on Bioinformatics and Bioengineering (BIBE 2012), Cyprus (pp 677-682).

24. Groumpos, P. P. \& Gkountroumani, V. (2014). A New Control Strategy for Modelling Wind Systems Using Fuzzy Cognitive Maps, Journal of Energy and Power Engineering, 8(11), David Publishing Company, Inc.

25. Groumpos, P. P. (2014). Intelligent Decision Making Support Systems: Issues and Challenges. In Proc. of International Conference Information Technologies for Intelligent Decision Making Support, May 2014, Ufa, Russia (pp. 25- 34).

26. Groumpos, P. P. (2016). Deep Learning vs. Wise Learning: A Critical and Challenging Overview. In IFAC Intern. Conf. on International Stability, Technology and Culture, TECIS 2016, Durres, Albania.

27. Groumpos, P. P. (2017). Intelligent Control and Cognitive Control for global coherence and sustainable growth for the humankind. In Plenary paper presented at the Inter. Confer. IFAC TECIS 2018, Baku.

28. Jang, R. J-S. (1997). Fuzzy Inference Systems, Chapter 4, 73-91.

29. Kazdin, A. E. \& Wilson, G. T. (1978). Evaluation of behavior therapy: Issues, evidence, and research strategies. Cambridge, MA Ballinger.

30. Kosko, B. (1986). Fuzzy cognitive maps, International Journal of man-machine studies, 24(1), 65-75.

31. Levin, S. A. (2003). Complex adaptive systems: Exploring the known, the unknown and the unknowable, Bulletin of the American Mathematical Society, 40, 3-19.

32. LHuillier, S. A. J. (1861). Memoire sur le polyedrometrie, Annales de Mathematiques, 3, 169-189.

33. Mamdani, E. H. \& Assilian, S. (1975). An experiment in linguistic synthesis with a fuzzy logic controller, International Journal of Man-Machine Studies, 7(1), 1-13.

34. Mesarovic, M., Macko, D. \& Takahara, Y. (1970). Theory of hierarchical multilevel systems. New York: Academic Press. 
35. Miller, G. A. (2003). The cognitive revolution: a historical perspective, $J$. Trends in Cognitive Sciences, 7(3), 141-144. March 2003, Elsevier.

36. Mpelogianni, V. G. \& Groumpos, P. P. (2015). Using fuzzy control methods for increasing the energy efficiency of buildings, International Journal of Monitoring and Surveillance Technologies Research (IJMSTR), 3(4), 1-22.

37. Mpelogianni, V. G. \& Groumpos, P. P. (2016). A revised approach in modeling fuzzy cognitive maps. In $24^{\text {th }}$ Mediterranean Conference on Control and Automation (MED), Athens (pp. 350-354). IEEE.

38. Mpelogianni, V. G. \& Groumpos, P. P. (2016). Towards a new approach of fuzzy cognitive maps. In 7th International Conference on Information, Intelligence, Systems \& Applications, Chalchidiki (pp.1-6). IEEE.

39. Mpelogianni V. G. \& Groumpos, P. P. (2018). Re-approaching fuzzy cognitive maps to increase the knowledge of a system, $A I \&$ SOCIETY, 1-14.

40. Nelson, R. J. (1976). Structure of Complex Systems, Philosophy of Science Association, 2, 523-542.

41. Papageorgiou, E. I., Stylios, C. D. \& Groumpos, P. P. (2004). Active Hebbian learning algorithm to train fuzzy cognitive maps, International Journal of Approximate Reasoning, 37, 219-249.

42. Papageorgiou, E. I. \& Stylios, C. D. (2008). Fuzzy cognitive maps, Handbook of Granular Computing, 755-774. John Wiley \& Son Ltd, Publication Atrium, England.

43. Papageorgiou, E. I. (2011). Review study on Fuzzy Cognitive Maps and their applications during the last decade. In 2011 IEEE International Conference on Fuzzy Systems, Taipei, Taiwan (pp. 828-835).

44. Pfluger, N., Yen, J. \& Langari, R. (1992). A defuzzyfication strategy for a fuzzy logic controller employing prohibitive information in command formulation. In Proceedings of IEEE Intern. Confer. in Fuzzy Systems. San Diego (pp. 717-723).

45. Saade, J. J. (1996). A unifying approach to defuzzification and comparison of the outputs of fuzzy controller, IEEE Trans. Fuzzy Syst., 4, $227-237$.

46. Stylios, C. D. \& Groumpos, P. P. (2004). Modeling complex systems using fuzzy cognitive maps, IEEE Transactions on Systems, Man and Cybernetics, Part A: Systems and Humans, 34(1), 155-162.

47. Taber, W. R. (1991). Knowledge processing with fuzzy cognitive maps, Expert Systems with Applications, 2(1), 83-87.

48. Vergini, S. E. \& Groumpos, P. P. (2017). New concerns on fuzzy cognitive maps equation and sigmoid function. In Proc. of $25^{\text {th }}$ Mediterranean Conference on Control and Automation MED 2017, Malta (pp. 11131118). IEEE Conference.

49. Vergini, S. E. \& Groumpos, P. P. (2018). Modeling Nearly Zero Energy Building Using New Equations and Separating Concepts on Fuzzy Cognitive Maps. In Proc. of IFAC TECIS 2018, September 13-15, Baku, Azerbaijan.

50. Winograd, T. (1982). Language as Cognitive Process, Volume 1, Syntax, Addison-Wesley.

51. World Economic Forum (2017). The global risks Report 2017, Annual Report.

52. Yu, W. (Ed.) (2009). Recent Advances in Intelligent Control System. Springer.

53. Cai, Z.-X. (1997). Intelligent Control: Principles, Techniques and Applications. Book Series: Series in Intelligent Control and Intelligent Automation, 7, World Scientific.

54. Zadeh, L. A. (1984). Making computer think like people, IEEE Spectrum, 21, 26-32. 